
pré-operatória de enfermagem sobre a ótica dos enfermeiros do centro-cirúrgico de um hospital universitário. Revista Eletrônica de Enfermagem, v. 06, n. 02, p. 213-222, 2004. Disponível em www.fen.ufg.br

\title{
ESTUDO DA VISITA PRÉ-OPERATÓRIA DE ENFERMAGEM SOBRE A ÓTICA DOS ENFERMEIROS DO CENTRO-CIRÚRGICO DE UM HOSPITAL UNIVERSITÁRIO.
}

\author{
A STUDY OF PRE OPERATION NURSING VISIT ABOUT THE NURSES' VIEW FROM THE SURGERY \\ ROOM OF A UNIVERSITY HOSPITAL.

\section{ESTUDIO DE LA VISITA PRE-OPERATORIA DE ENFERMÉRIA ECHA SOB LA MIRADA DE LOS ENFERMEROS DEL CENTRO QUIRURGICO DE UN HOSPITAL UNIVERSITÁRIO.}

\section{Giovanna Vallim Jorgetto ${ }^{1}$ Rachel Noronha ${ }^{2}$ Izilda Esmenia Muglia Araújo ${ }^{3}$}

\begin{abstract}
RESUMO - Este estudo objetivou realizar uma análise da importância da VPOE, segundo enfermeiros do Centro Cirúrgico de Rotina de um Hospital Universitário; e aplicar o instrumento de comunicação escrita na VPOE, proposto por ARAUJO \& NORONHA (1995). Os resultados obtidos foram: 92,9\% dos enfermeiros do Centro Cirúrgico de Rotina julgam importante a realização da VPOE para o paciente e assistência de Enfermagem e 85,7\% julgam ser a VPOE importante para o enfermeiro que trabalha em Centro Cirúrgico. O instrumento de comunicação escrita na VPOE foi aplicado com sucesso, sendo este de fácil preenchimento com perguntas claras, demonstrando ser, portanto bastante viável, porém itens do instrumento como Tipo Sanguíneo e Fator RH não conseguiram serem preenchidos mesmo após verificação dos prontuários. Assim sendo, os resultados permitem sugerir a implantação do instrumento de comunicação escrita proposto por ARAÚJO \& NORONHA (1995) neste Hospital Universitário propondo, porém inclusão nos prontuários dos pacientes de dados sobre Tipo Sanguíneo e Fator RH.
\end{abstract}

PALAVRAS-CHAVE: Enfermagem de Centro Cirúrgico; Enfermagem Perioperatória; Cuidados de Enfermagem.

\begin{abstract}
This study to do an analysis of the PONV`s importance, by nurses from the Daily's Surgery Room of an University Hospital, through forms distributed to them and to apply the written communication instrument on the PONV, proposed by NORONHA \& ARAÚJO (1995). The results this research were: 92,9\% of the nurses from the Daily's Surgery Room think that it is important the performance of the PONV to the patient and Nursing aid, and $85,7 \%$ think the PONV is important for the nurse who works in a Surgery Room. The written communication instrument on the PONV was applied with success, being really easy to fill it in with clear questions, showing so to be a lot of viable but some items of the instrument like blood group and FATOR RH couldn't be filled even after the records check. In this way, I think it is worth the suggestion off sitting the written communication instrument proposed by ARAÚJO AND NORONHA (1995) at this State University, proposing however, inclusion on the patients' records data about blood group and FATOR RH.
\end{abstract}

KEYWORDS - Operating Room Nursing; Perioperative Nursing; Nursing Care.

RESUMEN - Este estúdio objetivó realizar una análise de la importancia de la Visita Pre-Oquirpurgica de Enfermeira (VPOE), de acuerdo con los enfermeros de el centro quirúrgco rutinero de en Hospital Universitario, y aplicar el instrumento de VPOE propuesto por ARAUJO \& NORONHA (1995). Los resultados obtenidos fueron $92 \%$ de los enfermeros que trabaja en el centro Quirúrgico. El instrumento de comunicacion escrita en la VPOE fue aplicado con exito, siendo renellado con facilidad, con perguntas objetivas, entretanto se demostrando viable, con todo, algunos espacios para seren rellenados del instrumento como: tipo sanguineo, factor RH no conseguiran serlo, mismo después dela verificación de pronturios. Luego los resultados permiten

\footnotetext{
${ }^{1}$ Enfermeira do Centro de Reabilitação de Casa Branca, Casa Branca-SP, netperna@uol.com.br

2 Enfermeira. Doutora em Enfermagem. Professora Assistente do Curso de Graduação em Enfermagem (Aposentada) da FCM - UNICAMP.

Enfermeira, Prof. Assist. Dra. do Curso de Graduação em Enfermagem da FCM- UNICAMP. iema@fcm.unicamp.br.
} 
seguir la comunicación escrita propuesto por ARAÚJO \& NORONHA (1995) en un Hospital Universitario, proponiéndo la inclusión en los prontuarios datos como tipo sanguineo y factor $\mathrm{RH}$.

TÉRMINOS CLAVES: Enfermería en Sala Quirúrgica; Enfermería Perioperatoria; Atención de Enfermeria.

\section{INTRODUÇÃO}

A pessoa que será submetida a uma cirurgia apresenta diversos temores que podem alterar seu equilíbrio.

Um contato dos profissionais de saúde, dentre eles, o enfermeiro, possivelmente poderá ajudar esta pessoa no sentido de fornecer-lhe informações e diminuir sua insegurança, conforme foi constatado por vários autores (SILVA, 1987; SAWADA, 1991, CASTELLANOS et al, 1984, BIANCHI et al, 1983, SALZANO, 1983, KAMIYAMA, 1972)

Desta forma, manter um contato prévio com a pessoa que se submeterá a uma cirurgia representa um momento muito importante, uma vez que a explicação sobre os procedimentos a que este paciente será submetido seria de grande ajuda para diminuir seus temores, sua insegurança, e a apreensão por ele sentida.

Este procedimento recebe o nome de Visita Pré-Operatória de Enfermagem VPOE - e vem sendo realizada no Brasil desde 1975, data em que surge a primeira divulgação formal a respeito (CASTELLANOS et al, 1984).

Para o paciente, o período denominado pré-operatório é de vital importância em nível bio-psico-sócioespiritual, desencadeando a ansiedade, os temores (da cirurgia, da dor, de não voltar da anestesia, da morte) e o medo do desconhecido em geral (BIANCHI et al, 1983).

Neste período é que a assistência de enfermagem se faz extremamente importante, com o objetivo de preparar o físico e principalmente o emocional do paciente, preocupando-se com seu eu e com o que ocorrerá com seu corpo (BIANCHI et al, 1983).

Preocupados com este aspecto da assistência de enfermagem, vários estudiosos do assunto (SILVA, 1987; BIANCHI et al, 1983; SALZANO, 1983; SAWADA, 1991; SANTOS et al, 1981) incluem como responsabilidade da enfermeira que atua no centro cirúrgico (CC) a realização da VPOE, que tem como objetivos gerais esclarecer possíveis dúvidas que o paciente possa vir a ter, assim como seus familiares, diminuindo sua ansiedade e medo e dessa forma preparar uma melhor assistência de enfermagem nos períodos trans e pós-operatórios. Assegurando sua individualidade, além de promover a continuidade dos cuidados de enfermagem e favorecer uma interação muito importante entre o enfermeiro e o paciente.

No entanto, apesar da importância desta fase dentro do processo de assistência ao paciente, os instrumentos de comunicação escrita de enfermagem utilizados na VPOE parecem não estar sendo adotados de forma efetiva, uma vez que não foram encontrados na literatura relatos de seu uso contínuo, fato que torna relevante a busca de pesquisar soluções referentes a sua operacionalização no cotidiano do enfermeiro.

Diante do exposto e pelo interesse em aprofundar o assunto, o presente trabalho pretende analisar a importância da VPOE, do ponto de vista dos enfermeiros do centro cirúrgico de um hospital universitário, bem como testar o instrumento de comunicação escrita de enfermagem, proposto por Araújo e Noronha (ARAÚJO et al, 1996).

\section{OBJETIVOS}

- Verificar a importância da Visita PréOperatória de Enfermagem (VPOE), segundo os enfermeiros do Centro Cirúrgico de Rotina de um Hospital Universitário do Estado de São Paulo. 

pré-operatória de enfermagem sobre a ótica dos enfermeiros do centro-cirúrgico de um hospital universitário. Revista Eletrônica de Enfermagem, v. 06, n. 02, p. 213-222, 2004. Disponível em www.fen.ufg.br

- Testar o instrumento de comunicação escrita de Enfermagem na VPOE, proposto por ARAÚJO \& NORONHA (1996).

- Oferecer subsídios para a operacionalização da VPOE, com base na análise dos resultados obtidos.

\section{METODOLOGIA}

O presente trabalho, na sua fase de coleta de dados, foi dividido em duas partes. $\mathrm{Na}$ primeira foram entrevistados todos os enfermeiros dos três turnos de trabalho (manhã, tarde e noite) que atuam no Centro Cirúrgico de Rotina (CCR) de um Hospital Universitário Governamental do Estado de São Paulo, durante o período de trinta dias. Foi utilizado nesta entrevista um formulário semi-aberto (Apêndice I) composto de três partes: 1ª) Apresentação da pesquisadora; $2^{\mathrm{a}}$ ) Características da população estudada e $3^{a}$ ) Investigação da importância da VPOE, com justificativas. Este instrumento de coleta de dados foi entregue à diretora do serviço de enfermagem, sendo que a supervisora de cada plantão (manhã, tarde e noite) se encarregou de distribuir e posteriormente recolher 0 instrumento devidamente preenchido pelas enfermeiras. Esta atitude foi tomada no sentido de garantir total anonimato nas respostas emitidas pelas enfermeiras e de não prejudicar $\mathrm{o}$ andamento do serviço das mesmas no Centro Cirúrgico, por ocasião da entrevista que seria feita em horário de serviço e haver um número reduzido de profissionais atuando neste setor (segundo informações das enfermeiras do C CR). $\mathrm{Na}$ segunda parte, foi aplicado o instrumento de comunicação escrita de enfermagem utilizada na VPOE (Anexo I), no qual foram alvo de desta pesquisa pacientes candidatos a cirurgias eletivas, cujos nomes constavam nas escalas diárias do CCR e que estavam em condições de comunicação verbais e maiores de dezoito anos. Para tanto, foram escolhidos de maneira aleatória, através de sorteio, dois pacientes por dia, na escala de cirurgias da manhã do dia seguinte.

As entrevistas foram realizadas no período da tarde, logo após o sorteio e, se o paciente não estivesse internado, ou não fosse encontrado na sua unidade de internação, haveria sorteio de outro paciente. Esta seqüência seria seguida até a obtenção de duas entrevistas diárias, sendo assim constituída a amostra do presente estudo. Os dados referentes a VPOE foram coletados nas enfermarias onde os pacientes estavam internados, obedecendo à escala de cirurgias do CCR do Hospital Universitário, durante quatro meses, de segunda a quinta-feira no período da tarde, com base nas escalas de cirurgia previamente consultadas, no intuito de sortear os integrantes do presente estudo.

\section{RESULTADOS E DISCUSSÃO}

A análise dos dados coletados foi efetuada em duas partes: uma que se refere à importância da Visita Pré-Operatória de Enfermagem segundo entrevista feita com os enfermeiros do Centro Cirúrgico de Rotina; e outra sobre a aplicação do instrumento de comunicação escrita de enfermagem na VPOE. Os dados obtidos foram tabulados manualmente pela aluna pesquisadora e posteriormente avaliados quantitativa e qualitativamente, com base na análise dos percentuais e nas respostas obtidas das entrevistas com os enfermeiros e pacientes, conforme discussão a seguir.

Todos os enfermeiros que atuam no CCR deste Hospital Universitário, onde a pesquisa foi realizada, são do sexo feminino.

Pode-se observar que a maioria dos enfermeiros $(57,2 \%)$ encontra-se na faixa etária de 30 a 34 anos. Outros quatro $(28,6 \%)$ estão na faixa etária de 34 a 36 anos, enquanto dois deles estão respectivamente nas faixas etárias de 24 a 30 anos e 36 a 40 anos.

Desta população de enfermeiros estudada, $71,4 \%$ deles encontram-se 
formados de nove a 13 anos, dois (14,3\%) estão formados num período que variou de cinco a nove anos e dois enfermeiros estão cada um deles respectivamente formados há menos de um ano e de um a cinco anos. Observou-se que oito enfermeiros $(57,2 \%)$ têm de seis a nove anos de trabalho no CCR, onde a pesquisa foi realizada; três $(21,4 \%)$ trabalham há menos de um ano, dois $(14,3 \%)$ trabalham de um a três anos e apenas um enfermeiro está trabalhando neste local num período que varia três a seis anos.

Apenas três enfermeiros (21,4\%) ocupam cargo de chefia e este dado devese ao fato de haver uma supervisora ou chefe do serviço de enfermagem por plantão, e ser o CCR dividido em três plantões (manhã, tarde e noite). Os demais 11 enfermeiros $(78,6 \%)$ atuam somente na área assistencial sendo que apenas dois $(14,3 \%)$ têm curso de especialização em CC. Os que trabalham sem terem especialização contam apenas com a disciplina de Enfermagem em Centro Cirúrgico que cursaram na época da graduação e a experiência profissional adquirida ao longo dos anos, visto que a maioria deles $(57,2 \%)$ tem mais de seis anos de trabalho em centro cirúrgico.

Os enfermeiros deste $\mathrm{CC}$, onde a pesquisa foi realizada, tiveram um consenso com relação às respostas emitidas sobre 0 assunto e, a maioria $(85,7 \%)$, julga ser a VPOE importante para o paciente cirúrgico, pelo fato do mesmo ser esclarecido quanto ao procedimento anestésico-cirúrgico ao qual irá se submeter, além de ficar conhecendo um dos membros da equipe que irá recebê-lo no CC. Estas atitudes contribuem muito para a diminuição da ansiedade, medo e estresse do paciente. Apenas um enfermeiro $(7,1 \%)$ respondeu não considerar importante a VPOE para o paciente cirúrgico, alegando que o paciente já passa diversas vezes pela Visita PréAnestésica, por ser neste Hospital
Universitário comum a suspensão de cirurgias, além de ter que responder praticamente às mesmas questões na VPOE, fato que segundo este enfermeiro torna-se bastante cansativo.

Verificou-se que $85,7 \%$ dos enfermeiros julgam ser a VPOE importante para o enfermeiro que atua no $\mathrm{CC}$ e as justificativas apresentadas foram as que esta visita permite ao enfermeiro conhecer melhor o paciente antes da cirurgia levantando suas necessidades e transmitindo confiança e segurança aos mesmos; desenvolver tarefas específicas do enfermeiro; além do relacionamento interpessoal com 0 paciente satisfazer profissionalmente o enfermeiro. Por sua vez, dois enfermeiros (14,3\%) não julgam a VPOE importante para o enfermeiro que atua em Centro Cirúrgico, alegando este não ter tempo para a realização da visita, além de serem os cuidados de enfermagem no período pós-operatório baseados em orientações médicas.

Foram entrevistados 62 pacientes, que fizeram parte do sorteio aleatório, candidatos a cirurgias eletivas, no período de quatro meses (abril a julho) com predominância do sexo masculino. A maioria (14,5\%) encontrava-se na faixa etária de 43 a 48 anos e o paciente mais velho tinha 83 anos. As faixas etárias de menor incidência foram as de 28 a 33 anos e 33 a 38 anos com um número de dois pacientes entrevistados em cada uma delas, conforme consta na tabela 1.

A amostra foi constituída de pacientes internados nas enfermarias de cirurgia vascular, cardíaca, do trauma, torácica e cabeça e pescoço; sendo que houve uma ocorrência maior de casos cirúrgicos na faixa etária que compreende 68 a 73 anos, tendo nove pacientes do sexo masculino e quatro do sexo feminino. Além disso, demonstrou um certo equilíbrio na sua distribuição total (tabela 1). 

pré-operatória de enfermagem sobre a ótica dos enfermeiros do centro-cirúrgico de um hospital universitário. Revista Eletrônica de Enfermagem, v. 06, n. 02, p. 213-222, 2004. Disponível em www.fen.ufg.br

TABELA 01- Distribuição dos pacientes internados nas diferentes Unidades de Internação, segundo o sexo e faixa etária, Campinas-SP.

\begin{tabular}{l|l|l|l|l|l|l|l|l|l|l|l}
\hline $\begin{array}{l}\text { Especialida } \\
\text { des }\end{array}$ & \multicolumn{2}{l|}{$\begin{array}{l}\text { Cirurgia } \\
\text { cardíaca }\end{array}$} & \multicolumn{2}{l|}{$\begin{array}{l}\text { Cirurgia } \\
\text { vascular }\end{array}$} & \multicolumn{2}{l|}{$\begin{array}{l}\text { Gastrocir } \\
\text { urgia }\end{array}$} & \multicolumn{2}{l}{$\begin{array}{l}\text { Cirurgia } \\
\text { do } \\
\text { trauma }\end{array}$} & \multicolumn{2}{l}{$\begin{array}{l}\text { Cirurgia } \\
\text { cabeçal } \\
\text { pescoço }\end{array}$} \\
\hline $\begin{array}{l}\text { Faixa } \\
\text { Etária }\end{array}$ & M & F & M & F & M & F & M & F & M & F \\
\hline $18-23$ & 2 & 1 & 1 & & & & 2 & 2 & & \\
\hline $23-28$ & & & & & & & 1 & & & 1 \\
\hline $28-33$ & & & & & 1 & 1 & & & & \\
\hline $33-38$ & & & & & & 1 & 1 & & & \\
\hline $38-43$ & 1 & & & & & & 1 & & & \\
\hline $43-48$ & 1 & 1 & 1 & 1 & 1 & 2 & 1 & & & \\
\hline $48-53$ & & 1 & & & & & 1 & & & \\
\hline $53-58$ & 1 & & & & & & & & & \\
\hline $58-63$ & & 1 & 1 & 2 & & & & & & \\
\hline $63-68$ & 1 & & 3 & & & & 1 & & & \\
\hline $68-73$ & 1 & & 1 & 4 & & & & & & \\
\hline $73-78$ & 1 & 1 & 1 & & & & & 2 & & \\
\hline $78-83$ & & & 1 & 1 & & & & & & \\
\hline$* \mathrm{M}=$ masculino/ F= feminino & & & & & &
\end{tabular}

Dos $10(16,1 \%)$ entrevistados que apresentaram algum tipo de alergia 0 agente causador mais freqüente foi a Penicilina, seguido posteriormente em igual escala por outros fármacos como Bromudato de fenoterol, Cloridrato de buflomedil, Sulfazolamidas, Ácido Acetil Salicílico e Lidocaína; além de outros agentes diversos como algodão, detergente, alimentos com $\mathrm{pH}$ ácido $\mathrm{e}$ peixes de um modo geral. Todos esses casos encontravam-se devidamente identificados na cabeceira do paciente a fim de se evitar possíveis intercorrências. Esta atitude demonstra existir neste hospital, uma assistência de enfermagem bastante preocupada com a integridade e segurança do paciente.
A maioria dos pacientes que fizeram parte desta pesquisa (34 / 54,9\%) não apresentava antecedentes prévios de cirurgia o que pode demonstrar uma falta de conhecimento do procedimento anestésicocirúrgico, e a necessidade de assistência de enfermagem no sentido de oferecer-lhe informações.

Como a maioria dos pacientes deste estudo possuía idade avançada e muitos deles apresentaram algum tipo de problema cardiovascular como, por exemplo, a hipertensão arterial que aparece em um número expressivo da amostra, o eletrocardiograma (ECG) se faz importante sendo, portanto bastante solicitado, conforme demonstra a tabela 2.

TABELA 02- Pacientes entrevistados que tiveram realizado um eletrocardiograma (ECG) datado após sua internação, Campinas-SP. 


\begin{tabular}{lll}
\hline ECG & No. & \% \\
\hline SIM & 53 & 85,5 \\
NÃO & 09 & 14,5 \\
\hline TOTAL & $\mathbf{6 2}$ & $\mathbf{1 0 0 , 0}$ \\
\hline
\end{tabular}

Todos os entrevistados deste estudo, possuíam uma radiografia datada após sua internação e este dado pode ser explicado devido ao tipo de patologias dos mesmos, e das unidades onde encontravam internados (cirurgia cardíaca, vascular, do trauma, torácica, cabeça/pescoço e gastrocirurgia), sendo a radiografia um exame complementar de diagnóstico necessário e, portanto bastante solicitada pela equipe médica.

Foram previamente consultados os prontuários, a fim de que fossem preenchidos os itens constantes no instrumento de comunicação escrita da VPOE, principalmente aqueles sobre os quais 0 paciente não apresentava conhecimento. Porém, dados como tipo sanguíneo e fator $\mathrm{RH}$, na grande maioria das vezes, não foram encontrados, mesmo após verificação dos prontuários. Apenas os exames sanguíneos de hematócrito $(\mathrm{Ht})$ e hemoglobina $(\mathrm{Hb})$ constavam nos mesmos.

Quanto à qualidade da assistência de enfermagem recebida nos procedimentos anestésico-cirúrgicos $50 \%$ dos pacientes entrevistados classificaram como boas e $25 \%$ classificaram como regulares. Uma porcentagem de pacientes $(10,7 \%)$ julgam como ruins e apenas $14,3 \%$ do total de entrevistados classificam estes procedimentos como ótimos.

TABELA 03 - Classificação segundo a percepção dos pacientes entrevistadosquanto a qualidade da assistência recebida nos procedimentos cirúrgicosprévios. Campinas-SP.

\begin{tabular}{lcc}
\hline CLASSIFICAÇÃO & No. & $\%$ \\
\hline ÓTIMO & 04 & 14,3 \\
BOM & 14 & 50,0 \\
REGULAR & 07 & 25,0 \\
RUIM & 03 & 10,7 \\
\hline TOTAL & $\mathbf{6 2}$ & $\mathbf{1 0 0 , 0}$ \\
\hline
\end{tabular}

Em relação ao estado de nutrição e hidratação dos pacientes entrevistados, foi notado que a maioria deles $(26,6 \%)$ apresentaram mucosas ressequidas $\mathrm{e}$ outros apresentaram problemas não mencionados neste item do instrumento, tais como: palidez (dois entrevistados ou $2,1 \%$ do total), inapetência (dois entrevistados ou $2,1 \%$ do total), icterícia (três entrevistados ou 3,2\% do total), e por último a presença de 13 entrevistados ou $13,8 \%$ do total em bom estado de nutrição e hidratação. Além disso, observou-se que muitos se apresentavam emagrecidos $(21,3 \%)$ e apenas um deles apresentou astenia. 


\begin{tabular}{lcc}
\hline \multicolumn{3}{c}{$\begin{array}{l}\text { entrevistados em um Hospital Universitário do Estado } \\
\text { de São Paulo*, Campinas-SP. }\end{array}$} \\
\hline ESTADO DE NUTRIÇÃO E HIDRATAÇÃO & No. & $\%$ \\
\hline EMAGRECIDO & 20 & 21,3 \\
MUCOSAS RESSEQUIDAS & 25 & 26,6 \\
TURGOR DIMINÚIDO & 11 & 11,7 \\
SEDE & 08 & 8,5 \\
ASTENIA & 01 & 1,1 \\
EDEMA & 09 & 9,6 \\
OUTROS & 20 & 21,3 \\
\hline TOTAL & $\mathbf{6 2}$ & $\mathbf{1 0 0 , 0}$ \\
\hline
\end{tabular}

* Na tabela acima uma ou mais opções foram assinaladas para cada paciente, pelo fato destes apresentarem mais de um dos problemas acima descritos.

Os pacientes que apresentaram conhecimento anestésico-cirúrgico suficiente foram aqueles com antecedentes prévios de cirurgia. Os que apresentaram pouco conhecimento sobre o processo anestésico-cirúrgico $(24,2 \%$ do total) foram aqueles que seriam submetidos a cirurgias mutilantes, cirurgias com pós-operatório bastante complicado, ou retirada de neoplasias. Já os pacientes sem nenhum conhecimento sobre o processo anestésicocirúrgico foram os com grau de instrução baixo e/ou que tinham acabado de dar entrada na unidade de internação.

A condição da musculatura dos pacientes entrevistados apresentou a seguinte distribuição: hígida em 47 (75,8\%) e alterada em $15(24,2 \%)$.

A perda de massa muscular surge devido a diversos fatores, dentre os quais aqueles ligados à patologia que acomete 0 paciente. Além disso, deve ser levado em consideração, o fato deste hospital atender pessoas carentes e/ou em estado avançado de suas enfermidades, a exemplo das neoplasias malignas, encontrando-se geralmente em desnutrição severa, o que tem por conseqüência um peso abaixo do normal. (SANTOS et al., 1981).

Todos os pacientes entrevistados apresentaram rede venosa visível. Os pacientes que apresentaram rede venosa alterada foram submetidos à quimioterapia venosa (nove pacientes), processo este que torna as veias esclerosadas e difíceis de serem puncionadas. Outros pacientes com rede venosa alterada apresentaram veias entumecidas ("duras"), fato que pode ser associado a suas idades avançadas, entre 70 e 81 anos, totalizando três entrevistados (GUYTON, 1988).

Faziam uso contínuo de medicação 45(72,6\%) dos pacientes entrevistados. Este dado é subsidiário às cirurgias, pois através dele foi possível identificar o tipo de medicamento usado pelo paciente, bem como também fornecer dados para a análise de possíveis interações medicamentosas durante o ato anestésicocirúrgico. Pode ser constatado que os sistemas orgânicos mais freqüentemente acometidos por enfermidades nos pacientes entrevistados foram 0 respiratório, cardiovascular e gastrintestinal. Estes fatos encontrados podem ser explicados através das seguintes informações: a maioria dos pacientes entrevistados encontrava-se na faixa etária acima de 53 anos, os quais apresentam mais probabilidade de serem acometidos por diferentes enfermidades. Nesta faixa etária da maioria dos entrevistados, (classificada como $3^{a}$ idade), surgem com maior freqüência problemas no aparelho cardiovascular como, hipertensão arterial leve, moderada ou severa; no aparelho respiratório (problemas pulmonares diversos que 
levam a dispnéia aos médios esforços) e no aparelho gastrintestinal (principalmente a constipação, pelo fato da população brasileira de um modo geral, não possuir hábitos alimentares condizentes com uma dieta equilibrada além de ser esta pobre em fibras alimentares (KAMIYAMA, 1972). Já os pacientes que não apresentaram problemas nos sistemas orgânicos são aqueles pertencentes, em seu maior número, a faixas etárias mais jovens (18 a 33 anos).

TABELA 05- Impressões* de sentimentos percebidos pelo entrevistador, Campinas-SP.

\begin{tabular}{lcc}
\hline SENTIMENTOS & No. & $\%$ \\
\hline ISOLADO & 04 & 4,1 \\
AGITADO & 11 & 11,3 \\
CONFUSO & -- & -- \\
AGRESSIVO & 01 & 1,0 \\
ANSIOSO & 26 & 26,8 \\
APÁTICO & 13 & 13,4 \\
EUFÓRICO & 03 & 3,1 \\
CALMA APARENTE & 28 & 28,8 \\
OUTROS & 11 & 11,3 \\
\hline TOTAL & $\mathbf{9 7}$ & $\mathbf{1 0 0 , 0}$ \\
\hline
\end{tabular}

* Foram observadas mais de uma impressão de sentimentos por paciente entrevistado.

Nesta tabela pode-se constatar que não foi observada nenhuma impressão de confusão nos pacientes, pelo fato de todos os entrevistados neste estudo apresentarem como pré-requisito condições normais de comunicação verbal. Com relação aos sentimentos percebidos, 0 de calma aparente foi bastante evidenciado e este pode ser explicado por uma assistência de enfermagem eficiente ao nível das informações prestadas ao paciente, como também pelo fato de um grande número de pacientes entrevistados apresentar antecedentes prévios de cirurgia, o que sugere que os mesmos tinham noções dos períodos pré e pós-cirúrgicos. Outra impressão de sentimento bastante percebida pelo entrevistador e em número considerável foi à ansiedade, o que vem a demonstrar ser 0 ato anestésico-cirúrgico um desencadeador de desequilíbrio das necessidades bio-psico-socio-espirituais do paciente $^{3}$. Já nas impressões de sentimentos registradas como "outros", as mais freqüentes manifestações foram as de depressão e angústia, seguidas em menor número pelo nervosismo, que poderia ser momentâneo ou um estado considerado constante.

A maioria dos pacientes (83,9\%) encontrava-se sem deficiências e/ou limitações sejam elas físicas ou mentais. As deficiências/limitações apresentadas como mental corresponde a uma seqüela de acidente vascular cerebral, onde o paciente tem pequena dificuldade de comunicação e por isso sua entrevista foi considerada neste estudo. Das deficiências/limitações físicas que surgiram, as mais freqüentes são limitações decorrentes de dispnéia aos médios esforços, seguido em igual número por dificuldade de locomoção devido à angina aos médios esforços, pressão arterial elevada e dificuldade circulatória intensa com perda de artelhos o que contribui para uma dificuldade no equilíbrio corpóreo. 

pré-operatória de enfermagem sobre a ótica dos enfermeiros do centro-cirúrgico de um hospital universitário. Revista Eletrônica de Enfermagem, v. 06, n. 02, p. 213-222, 2004. Disponível em www.fen.ufg.br

Dados do paciente como peso e altura, auxiliam no processo anestésico do mesmo, pois através deles é possível calcular a quantidade de drogas necessárias a serem oferecidas durante todo o ato cirúrgico.

Os parâmetros de freqüência cardíaca, pressão arterial, freqüência respiratória e temperatura são subsidiários às cirurgias e a maioria dos pacientes entrevistados apresentou estes dados dentro de uma faixa considerada por autores, como normais (GUYTON, 1988).

Com relação ao item do instrumento de comunicação escrita da VPOE que questionam "problemas/doenças não abordados durante entrevista", foi possível detectar problemas e/ou doenças tais como tabagismo, etilismo, hipertensão arterial, infarto agudo do miocárdio (IAM), diabetes melitus (DM), hepatite e febre reumática, entre outros.

\section{CONCLUSÕES}

Tendo em vista os objetivos propostos neste estudo, pode-se concluir que:

$\diamond \quad$ Do total de enfermeiros respondentes que participaram desta pesquisa, 92,9\% julgam importante à realização da Visita Pré-Operatória de Enfermagem para o paciente em fase pré-operatória e para a assistência de enfermagem, sendo que apenas $7,1 \%$ responderam não julgar importante a realização da VPOE nos itens acima questionados.

Julgam importante, $85,7 \%$ dos enfermeiros, a realização desta visita para o enfermeiro que atua em centro cirúrgico enfatizando o relacionamento enfermeiro-paciente e a satisfação profissional sentida pelo mesmo. Sendo que $14,3 \%$ dos enfermeiros não acham importante para o enfermeiro de $\mathrm{CC}$ a realização da VPOE alegando nas respostas, a falta de tempo.

$\diamond \quad O$ fato de não ser o mesmo enfermeiro o responsável pelo paciente durante toda sua permanência no Centro Cirúrgico. Vale acrescentar que neste hospital onde a pesquisa foi realizada a VPOE não é efetuada pelos enfermeiros que atuam no Centro Cirúrgico.

$\diamond \quad O$ instrumento de comunicação escrita VPOE proposto por Araújo e Noronha $^{8}$ foi aplicado com sucesso entre os pacientes candidatos a cirurgias eletivas, sendo este de fácil preenchimento com perguntas claras e objetivas demonstrando, portanto ser bastante viável. Porém, itens do instrumento como tipo sanguíneo e fator $\mathrm{RH}$, sobre os quais os pacientes não apresentavam conhecimento, não conseguiram ser preenchidos, mesmo após verificação do prontuário dos mesmos, o que demonstrou falta de anotações adequadas da equipe médica e de enfermagem, visto serem estes dados bastante importantes para 0 paciente pré-cirúrgico.

$\diamond$ Julgou-se válida a sugestão de implantação do instrumento de comunicação escrita VPOE proposto por Araújo e Noronha (1996), neste Hospital Universitário onde a pesquisa foi realizada, apenas com a sugestão de inclusão nos prontuários dos pacientes de dados sobre tipagem sanguínea e fator $\mathrm{RH}$.

\section{REFERÊNCIAS BIBLIOGRÁFICAS}

ARAÚJO, I.E.M.; NORONHA, R. Proposta de comunicação em Enfermagem: Visita Pré-Operatória de Enfermagem. $48^{\circ}$. Congresso Brasileiro de Enfermagem. Livro de Resumos. São Paulo: ABEn, p.399.1995. BIANCHI, E.R.F.; CASTELLANOS, B.E.P. Considerações sobre a visita pré-operatória do enfermeiro da unidade de centro cirúrgico: resenha da literatura estrangeira. Rev Paul. Enf., v. 5, n.3, p.161-166. 1983. CASTELANOS, B. E. P. et al.. Visita préoperatória do enfermeiro da unidade de centro cirúrgico: marcos referenciais para seu ensino no curso de graduação de enfermagem. Rev Paul. Enf., v.4, n.1, p.1014. 1984. 
JORGETTO; Giovanna Vallim; NORONHA; Rachel; ARAÚJO; Izilda Esmenia Muglia - Estudo da visita pré-operatória de enfermagem sobre a ótica dos enfermeiros do centro-cirúrgico de um hospital universitário. Revista Eletrônica de Enfermagem, v. 06, n. 02, p. 213-222, 2004. Disponível em www.fen.ufg.br

GUYTON, A. C. Fisiologia humana. Rio de Janeiro. Ed: Guanabara Koogan. 1988.

SANTOS, E.S.; CABERLON, I.C. Visita pré e pós-operatória aos pacientes. Enfoque, v.6, n.9, p.41-45. 1981.

SALZANO, S.D.T. Instrumento de comunicação de enfermagem. Rev. Esc. Enf. USP, v.17, n.3, p.235-252. 1983.

SAWADA, N.O. A dimensão não verbal da interação enfermeiro-paciente em situação pré-operatória. Rev Paul. Enf., Número Especial, p.42-47. 1991.
SILVA, A. A visita pré-operatória de enfermagem pela enfermeira do centro cirúrgico. Rev. Esc. Enf. USP, v.21, n. 2, p. 145-160. 1987.

KAMIYAMA, Y. O doente hospitalizado e sua percepção quanto à prioridade de seus problemas. São Paulo, 1972, 111 p. Tese (Doutorado). Escola de Enfermagem da Universidade de São Paulo.

Texto recebido em 02/06/2004.

Publicação aprovada em 31/08/2004. 